\title{
On the Common Structure of the Primitive Ontology Approach and the Information-Theoretic Interpretation of Quantum Theory
}

\author{
Lucas Dunlap
}

\begin{abstract}
We use the primitive ontology framework of Allori et al. to analyze the quantum information-theoretic interpretation of Bub and Pitowsky. There are interesting parallels between the two approaches, which differentiate them both from the more standard realist interpretations of quantum theory. Where they differ, however, is in terms of their commitments to an underlying ontology on which the manifest image of the world supervenes. Employing the primitive ontology framework in this way makes perspicuous the differences between the quantum information-theoretic interpretation, and the various realist interpretations of quantum theory. It also allows us to identify a sense in which the commitments of quantum information-theoretic interpretation are underspecified. Several possible ways of completing the interpretation are presented, and it is suggested that the most likely strategy would leave the information-theoretic interpretation such that it would fail to qualify as a theory, according to the primitive ontology approach.
\end{abstract}

\section{Introduction}

A recent series of papers by Valia Allori has brought the conception of fundamental physical theories based on a primitive ontology to the attention of a broader audience in the philosophy of physics community. The primitive ontology framework (PO) has been well known in the debates about the interpretation of quantum mechanics since it was introduced by Allori's frequent collaborators in the 1990s (see [11] [12] [3] [4]). But Allori's recent clear presentation of PO (see [1] and [2]) has made it more amenable to broader application. In this paper, we will discuss the features of PO, and analyze the quantum-information theoretic interpretation - a competing interpretation of quantum theory-in its terms. The 
comparison between the two approaches is illuminating, particularly because PO provides a framework in which significant similarities can be seen between the two seemingly very different ways of conceiving of the quantum world.

The quantum information-theoretic interpretation of quantum theory (QIT) was fully formulated by Bub and Pitowsky [9], but had its origins in earlier work of Clifton, Bub, and Halvorson [10], Bub [7] and [8], and Pitowsky [15]. This interpretation is formulated fundamentally in terms of information-theoretic constraints on the possibility of correlations between events. It is argued that a small number of such constraints pick out the Hilbert space as the fundamental space in which the theory is formulated, which in turn imposes conditions on the possibilities of correlations between events. These information-theoretic principles have physical motivation, as they represent various "no-go" theorems of quantum mechanics. The interesting feature of QIT, though, comes in considering these kinematical principles to be the fundamental formulation of quantum theory. What quantum theory tells us about the structure of reality, according to this way of thinking, is that it conforms to a non-Boolean underlying event space, out of which an effectively classical macroscopic world can emerge.

It should be immediately obvious that there is some tension between this conception of the fundamental formulation of quantum theory, and the more straightforwardly realist approaches, including PO. However, we will argue that they are similar in that they both reject a central tenet of the various wave function ontology approaches. By analyzing QIT in terms of $\mathrm{PO}$, however, the ways in which they differ will also be clear. This analysis, we argue, helps bring to light an important sense in which the commitments of QIT are underspecified. Several possible ways to complete the interpretation are considered, and it is argued that the route seemingly most in line with the rest of Bub and Pitowsky's approach leaves QIT such that it would fail to qualify as a theory, according to PO.

\section{Primitive Ontology}

PO is a framework for the formulation of candidate fundamental physical theories. The most salient feature of PO is its insistence on clarity and perspicuity with respect to the ontological commitments of the physical theory. Each theory is explicit from the outset about its primitive ontology. That is, each proposed theory begins by stipulating what the theory is about. A theory must be about the behavior of localized material entities, on this view. In this respect, it is similar to Bell's focus on the "beables" of a theory (see [6] and [5]).

PO imposes additional constraints on the primitive ontology of a fundamental physical theory. Crucially, the elements of the primitive ontology must exist in three-dimensional physical space. It is only in this way that they can conceivably 
count as the fundamental constituents of everyday physical objects. The theory is therefore about the evolutions of, and interactions between, these entities, which gives rise to the manifest image of the world we experience.

The aim of a fundamental physical theory is, we believe, to describe the world around us, and in so doing to explain our experiences to the extent of providing an account of their macroscopic counterparts, an account of the behavior of objects in 3-space. Thus it seems that for a fundamental physical theory to be satisfactory, it must involve, and fundamentally be about, 'local beables' [...]. [3]

Therefore, in this framework, only certain of the interpretations of quantum theory that are usually considered to offer solutions to the measurement problem count as candidate fundamental physical theories. The most obvious fit with the PO framework is Bohmian Mechanics. Its primitive ontology-namely, particles with definite positions - is stipulated from the outset. Although the wave function is part of the ontology of the theory, it is secondary to the material particles.

$[\mathrm{E}]$ ven if the primitive ontology does not exhaust all the ontology, it is the one that makes direct contact between the manifest and the scientific image. Since the primitive ontology describes matter in the theory, we can directly compare its macroscopic behavior to the behavior of matter in the world of our everyday experience. Not so for the other non-primitive variables [including the wave function], that can only be compared indirectly in terms of the way in which they affect the behavior of the primitive ontology. [1]

The GRW theory can also be formulated in the PO framework, so long as

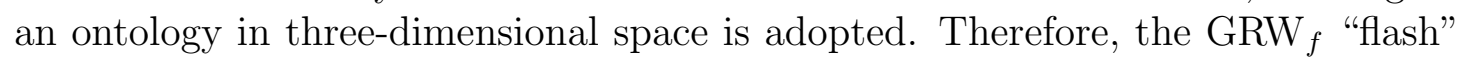
ontology version is consistent with PO, as is the $\mathrm{GRW}_{m}$ mass-density version.

The "bare" version of the theory, or $\mathrm{GRW}_{0}$, is not consistent with PO, since it holds that the wave function is the full story of the ontology of the world. In this case - as with all interpretations that take the wave function to be among the fundamental elements of the ontology - the space in which the wave function evolves in accord with the dynamics is $3 N$-dimensional configuration space. ${ }^{1}$

The problem of the correspondence between the ontology in the fundamental $3 N$-dimensional configuration space and the macroscopic three-dimensional world

\footnotetext{
${ }^{1}$ It is important to note that since the $\mathrm{GRW}_{0}$ theory does not include particles in threedimensional space in its picture of the world, the space in which the wavefunction evolves is not actually a configuration space of $N$ particles. However, the space on which the wave function is defined has a dimensionality equal to $3 N$, and is effectively identical to a configuration space of $N$ particles.
} 
of everyday experience has been well expressed elsewhere (see [13] and [14]), but it takes on an interesting character in the context of PO. The problem isn't simply one of correspondence between particular dimensions of the configuration space and the identity of particles in three-dimensional space. Rather, it is that, in the context of the PO, it is impossible for three-dimensional macroscopic objects to be made up of an ontology existing in an altogether different space. It is a gap in the ontology, not merely an underspecification of the correspondence between the two spaces. Allori et al. characterize such theories as ones "for which there exists no arrangement of stuff in physical three-dimensional space at all" [3].

This framework also rules out the Everett interpretation for the same reasons. However, Allori proposes an alternative version of the Everett interpretation that supplements the standard version with a primitive ontology. In order for the Everett interpretation to be formulable in PO, it needs to, like GRW, adopt either a "flash" or a mass-density ontology [1]. The "bare" version of the Everett interpretation (which Allori calls $\mathrm{MW}_{0}$ ) fails to be a candidate fundamental physical theory, because it does not have an ontology that exists in ordinary three-dimensional physical space.

The fact that PO is not consistent with the standard Everett interpretation shows that PO deviates in a significant way from the interpretations of quantum theory that have traditionally been taken to be realist. The difference can be located in the relationship presumed to hold between the mathematical structures of the theory and its ontology. The traditional scientific realist takes the mathematical objects in the theory to somehow represent elements of reality.

The following passage from David Wallace's The Emergent Multiverse is a suitable representative of the motivations of the realist wave function ontology position. He begins with a simple statement of scientific realism:

[W] hat scientific theories do is give us information about the the universewhat sort of things are in it, about how they are structured, about how they come into existence and interact and change and disappear. [16]

He then argues against those who would claim that quantum mechanics must be different in this respect:

$[\mathrm{N}]$ either the mathematical formalism of quantum mechanics, nor the standard conception of science is in any need at all of modification. Rather: the unmodified quantum theory can be taken as representing the structure of the world just as surely as any other theory of physics. [16]

What differs among the different standard realist interpretations is how much modification quantum theory must undergo in order to successfully represent the 
structure of the world and the fundamental entities in it. But what is shared among all of them is the idea that fundamental physical theories act as a guide to our understanding of what those entities and structures are. Physics is a guide to metaphysics on this conception. And in particular, each realist interpretation includes some commitment to what the fundamental ontology of the world is. The wave function corresponds, in these interpretations, to an element of reality.

I argue that this is the major point of departure for PO. As Allori emphasizes in [2], the primitive ontology approach is partially constitutive of a different understanding of what fundamental physical theories are, and how they function. The primitive ontology approach does not consider the ontology of the world to be determined by the structures in the mathematical formulation of the theory.

The starting idea is that when a scientist proposes a fundamental physical theory, she already has in mind what the theory is fundamentally about: the primitive ontology. This is the metaphysical role of the primitive ontology, it tells us what the world is made of according to the theory. [2]

With respect to the idea that the mathematical objects of the theory represent entities or structures in the world, she says

$[\mathrm{T}]$ here is already a natural interpretation for each mathematical object, namely, the one the proponent of the theory intended to give them! The scientists choice of what physically exists in the world will more or less automatically determine the mathematical object to represent it. [2]

Unlike the traditional realist interpretations, the ontology of the theory is not determined by its mathematical structure, and therefore the theory itself is not a guide to the underlying metaphysics. Rather, the ontology is determined at the outset by the author of the theory. She argues that the wave function ontology picture gets this backward.

$[\mathrm{T}]$ he wave function ontology view is misguided: it assumes the fact that the mathematical formalism of a theory can be interpreted a posteriori, whereas it was fixed a priori by the physicist when she formulated the theory. [2]

The emphasis of PO is that fundamental physical theories are not to be understood as a guide to fundamental ontology of the world. Rather, it holds that the way to conceive of a fundamental physical theory is as an account of how a given posited ontology underwrites, or causes, or explains the behavior of observable objects. 
Allori presumably doesn't consider this framework to amount to a demotion of the importance of the ontology of the theory. This is evidenced by her claim that differing primitive ontologies amount to the scientist offering a "metaphysical hypothesis" around which the theory is developed [2]. There is still space on this account to believe that there is a connection between the primitive ontologies of our best theories and entities and structures in the world.

However, PO does allow one the leeway to prefer certain proposed primitive ontologies for extra-theoretical reasons. For example, Allori insists that the primitive ontology for any candidate quantum theory be entities that exist in threedimensional space.

A primitive ontology in the familiar three-dimensional space evolving in time (or a space-time primitive ontology) is the natural metaphysical choice, if the theory with such a primitive ontology can be empirically and explanatorily adequate. [2]

She goes on to say

$[\mathrm{T}]$ hey are quantum theories in which, as in classical theories, there is stuff in space-time, and we can develop a clear explanatory scheme, along the lines of the classical one, to account for the macroscopic world. [2]

This focus on a theory being fundamentally understood as an account of a posited ontology underwriting the manifest image is one similarity that PO shares with QIT. Neither conceives of fundamental physical theories as giving guidance to metaphysics. Rather, they conceive of fundamental physical theories to primarily be about accounting for how the macroscopic manifest image supervenes on the quantum world.

\section{Quantum Information-Theoretic Interpretation}

The quantum information-theoretic interpretation of quantum theory is the outgrowth of results from two independent lines of inquiry of Bub and Pitowsky. Bub's earlier work in collaboration with Clifton and Halvorson [10], from which the CBH theorem resulted, forms the foundation of the interpretation. He later expanded on the implications of the theorem in [7] and [8]. In those papers, he invoked a distinction, due to Einstein, between principle and constructive theories, to argue that the fundamental formulation of a physical theory need not make reference to an underlying ontology and dynamics.

Pitowsky's argument from [15] introduced the idea of reconceptualizing quantum theory as being about the constraints imposed on the correlations between 
events implied by the Hilbert space formalism. The combination of the two projects allowed them to formulate a full-fledged interpretation of quantum theory that offered a solution to the measurement problem [9].

The $\mathrm{CBH}$ theorem was a breakthrough in the quantum information literature, because it seemed to offer a way of understanding the content of quantum theory based on physically plausible principles. These three principles - No Signaling, No Broadcasting, and No Unconditionally Secure Bit Commitment-when suitably translated into information-theoretic constraints on the probabilities of outcomes of experiments on isolated systems, come very near to uniquely picking out quantum theory from among a general space of possible probabilistic theories.

In [7] and [8], Bub argued that the $\mathrm{CBH}$ theorem shows that quantum theory is really a theory about the possibility of representing and manipulating information. If information is taken as a new physical primitive, then the three informationtheoretic constraints of the $\mathrm{CBH}$ theorem suggest a path to understanding quantum theory as a principle theory, which has no need for reference to an underlying ontology.

The question: What is information in the physical sense (if its not about the properties of physical stuff)? should be seen as like the question: What is a field in the physical sense (if it is not the vibration of a physical medium)? The answer is something like this: Quantum mechanics represents the discovery that there are new sorts of information sources and communication channels in nature (represented by quantum states), and the theory is about the properties of these information sources and communication channels. [7]

It is possible, he argues, to give a mechanical story, based on ontological primitives and dynamics, but it can have no additional empirical content over the principletheoretic formulation.

Pitowsky's 2006 paper [15] argues that quantum theory should be thought of as a new theory of probability. Like classical probability theory, it consists of a space of possible events, and a measure over it. In the quantum case, the space of possible events is identified with the lattice of the closed subspaces of Hilbert space, which Pitowsky says "represents the elements of reality in the theory". Pitowsky argues that conceiving of quantum theory in this way frees one from having to worry about the measurement problem as it is usually formulated. There is no possibility of explaining the particular outcome of an experiment on this view. The event space defines the possible outcomes of measurements, and the quantum state is taken to be an epistemic feature of the theory, encoding the experimenter's beliefs. Measurement outcomes - macroscopically observable outcomes of experimentsare taken as basic elements of the theory. These outcomes are systematic-meaning that the same measurements on similarly prepared systems will produce a stable 
set of outcomes - and are consistent with the structure of the closed subspaces of Hilbert space.

In "Two Dogmas About Quantum Mechnics", Bub and Pitowsky unify the elements of their previous work to create QIT. They begin by identifying the two assumptions they consider to be responsible for the widely-held belief that the quantum measurement problem has not been decisively solved. The two assumptions are (1) that measurement should never be included as an unanalyzable primitive in a fundamental physical theory, and (2) that the quantum state has some ontological significance as a truthmaker for propositions about the occurrence or non-occurrence of events. They argue that the rejection of these dogmas allows them to divide the measurement problem into two parts. The "big" measurement problem is the problem of giving a dynamical explanation of why particular measurements have particular definite outcomes. The "small" measurement problem is the problem of explaining how the seemingly classical macroworld in underwritten by the non-Boolean quantum event space. They claim to show that they can dismiss the former as a pseudo-problem, and they can solve the latter [9].

The argument against the need to address the "big" measurement problem proceeds as an argument from analogy. They argue that the Everett interpretation gets around explaining why particular experiments have particular definite outcomes by denying that experiments have definite outcomes at all. They identify the elements of the Everett interpretation that they believe qualify it as a realist interpretation that solves the measurement problem. They then argue that QIT shares all of these features.

The elements of the Everett interpretation that justify the claim that it solves the measurement problem are as follows: (1) a weighted structure of effectively classical worlds, which emerge via decoherence, (2) an argument that agents can be uncertain and have different preferences about futures states of the world, and (3) a claim that the agents' preferences should converge on the weights of the branches. These three elements together succeed in "saving the appearances" of a classical macroscopic reality.

QIT, while maintaining that experiments have particular outcomes, denies that a dynamical explanation of those facts is fundamental. However, by demonstrating a similarity between QIT and the Everett interpretation with respect to these three points, they argue that QIT has equal claim to being a realist interpretation with a solution to the measurement problem. They argue that a solution to the "big" measurement problem is not necessary to be considered a realist interpretation. Point (1) is the "small" measurement problem, which Bub and Pitowsky solve in a similar way to the Everett interpretation, via decoherence. Point (2) is a feature of QIT, since they take the quantum state to represent the epistemic situation of the experimenter. Point (3) is achieved by using Gleason's theorem. 
They appeal to Einstein's distinction between principle and constructive theories, and argue that the traditional realist interpretations are all to be understood as constructive versions of quantum mechanics, whereas their account is a principle version. They take the fact Einstein's principle account of Lorentz covariance (special relativity) was preferred to Lorentz's own constructive account to be encouraging evidence that a realist principle physical theory can supplant a constructive version. The fact that there was a possible dynamical explanation of Lorentz covariance (namely, Lorentz's own dynamical account) was taken to justify interpreting special relativity realistically.

Similarly, quantum mechanics has the resources to explain the outcome of any particular experiment dynamically. The process of decoherence ensures that the interactions that give rise to entangled states comprised of quantum systems and measuring devices will yield effectively classical macroscopic effects. That is to say, the dynamics of quantum mechanics is consistent with the classical probability space of macroscopic measurement outcomes.

This shows that the non-Boolean structure of the quantum event space is consistent with the effectively classical event space of macroscopic outcomes. However, the dynamical explanations of particular outcomes are not possible, since macroscopic events are taken as basic. Therefore, an account which supplements quantum mechanics with an underlying ontology, in order to give dynamical explanations, is less fundamental than QIT.

We argue that their focus on the "small" measurement problem as the more fundamental of the two is evidence of a certain parallel with the primitive ontology approach. The primary question for a physical theory on both conceptions is how it can underwrite the manifest image of the macroworld, not what metaphysical picture quantum theory requires us to accept about the elements of reality.

The traditional realist interpretations of quantum theory take the "big" measurement problem to be centrally important. Each of them offers a solution to it, and that solution crucially features a commitment to one fundamental ontology or another. Bohm's theory, for example, answers the "big" measurement problem by including particles with definite positions among the fundamental ontology, which determine the outcomes of experiments. It is the rejection of the idea that the theory needs an underlying metaphysical picture that allows Bub and Pitowsky to dismiss the "big" measurement problem.

\section{Common Structure in PO and QIT}

In this section, we will argue that there are two important similarities between PO and QIT, that set them both apart from the traditional realist wave function ontology approaches. The first is that they both reject the idea that the math- 
ematical objects in the theory should be seen as representing entities in reality. As discussed in Section 2, this standard scientific realist move is motivation for taking the wave function seriously as an element of reality. In both PO and QIT, however, they deny that the wave function is the right kind of mathematical object to possibly represent a fundamental enitity.

Of course, they both agree that there is something to be learned about the world from the mathematical features of quantum theory. In the PO case, Allori et al. suggest considering the wave function to be law-like, and to be a non-primary element of reality that is only discoverable by virtue of its action on the primitive ontology. The QIT case considers the structure of the Hilbert space to be the space of possible events. But in both cases, the mathematical objects of the theory are not a guide to the underlying ontology.

That is to say, both PO and QIT reject Bub and Pitowsky's second dogma, that the quantum state must be taken as representative of an element of physical reality. PO rejects the possible ontological fundamentality of the wave function. QIT rejects that the wave function could be an element of reality at all.

This is an explicit rejection of the wave function ontology approaches of the Everett interpretation and $\mathrm{GRW}_{0}$. PO holds that these are not candidate fundamental physical theories because they lack the right kind of ontology. QIT considers them to be non-fundamental because they add structure to quantum theory, without any gain in empirical content.

The second respect in which PO and QIT are similar is that they prioritize explanations of the emergence of the manifest image from the quantum world. On these views, the goal of a fundamental physical theory isn't as a guide to the underlying metaphysics of the world. Rather, its primary purpose is to give an explanation of the effectively classical macroscopic world of everyday experience. However, they differ on how to achieve this, and what would count as a successful theory with respect to this criterion.

PO argues the only way this can be accomplished is by telling a story of how the material entities that constitute macroscopic objects behave. Otherwise, the theory wouldn't be about anything at all.

What is wrong with $\mathrm{GRW}_{0}$, the bare version of GRW, which involves just the wave function and nothing else? Why does one need a PO at all? Our answer is that we do not see how the existence and behavior of tables and chairs and the like could be accounted for without positing a primitive ontology - a description of matter in space and time. [3]

QIT, on the other hand, holds that demonstrating the dynamical emergence of an effectively classical event space sufficiently underwrites the existence of the manifest image of everyday experience. 
The analysis shows that a quantum dynamics, consistent with the kinematics of Hilbert space, suffices to underwrite the emergence of a classical probability space for the familiar macro-events of our experience [...]. The explanation for such nonclassical effects as the loss of information on conditionalization is not provided by the dynamics, but by the kinematics, and given 'no cloning' as a fundamental principle, there can be no deeper explanation. In particular, there is no dynamical explanation for the definite occurrence of a particular measurement outcome, as opposed to other possible measurement outcomes in a quantum measurement process - the occurrence is constrained by the kinematic probabilistic correlations encoded in the projective geometry of Hilbert space, and only by these correlations. [9]

They argue that the existence of possible dynamical explanations of the outcomes of the experiments (including the wave function ontology and primitive ontology approaches, which they claim all add structure to the quantum formalism) serve as a proof of the consistency of their account. But it is important to note that these dynamical explanations are not fundamental.

\section{Comments}

The QIT denies the fundamentality of an underlying ontology, and is therefore open to the same critiques from the PO approach as the "bare" wave function ontology. Without an underlying ontology, it is difficult to understand what the theory claims macroscopic objects like tables and chairs are made of. In PO, the proposed ontology constitutes the objects of everyday experience, and so explains them. Bub and Pitowsky explicitly reject the notion that explanation of the state of a macroscopic system is necessary, but in so doing they seem to be demoting the idea of an underlying ontology altogether. And without a primitive ontology, QIT fails to qualify as a candidate fundamental physical theory, according to PO.

But QIT also claims to be a realist interpretation that solves the measurement problem. Perhaps there is an implicit ontology underwriting the view. After all, how could information be a fundamental element of reality if it was not being carried by some material entity? ${ }^{2}$ As they say

The possibility of a dynamical analysis of measurement processes consistent with the Hilbert space kinematic constraints justifies the informationtheoretic interpretation of quantum mechanics as realist and not merely

\footnotetext{
${ }^{2}$ It is worth nothing here that Bub and Pitowsky disavow the 'It-from-bit' position, which claims that immaterial information gives rise to the material world.
} 
a predictive instrument for updating probabilities on measurement outcomes. [9]

What elements of reality could the features of the theory be representing if not an underlying ontology?

I will consider four possible ways that QIT could be supplemented such that it would count as a candidate fundamental physical theory according to PO. The possible ways QIT could flesh out their ontology that I will consider are (1) a macroscopic ontology, where outcomes of experiments constitute the primitive ontology, (2) an event ontology, (3) a particle ontology in 3-d space, with an indeterministic dynamics, and (4) the principles that characterize the event space as the elements of reality.

With respect to option 1: It seems as though Pitowsky is making this claim in [15] by calling measurements, and measurement outcomes, unanalyzable. It seems that in that paper he holds that there is a definite distinction between the quantum and classical domains. Allori suggests that a position like this would be amenable to treatment in the PO framework.

It is interesting to note that even the orthodox quantum theory [...] involves such a dual structure: what might be regarded as its primitive ontology is the classical description of macroscopic objects, including in particular pointer orientations, while the wave function serves to determine the probability relations between the successive states of these objects. [2]

However, Pitowsky also claims in [15] that there are fundamental particles (see option 3 below), and neither of these positions seems to survive to the formulation of QIT in [9].

With respect to option 2: QIT seems to make this claim at times, as when they describe the kinematical constraints on correlations as being part of the fundamental formulation of the theory, and Hilbert space as a space of events. However, this would give rise to a correspondence problem - similar to the one encountered by the wave function ontology positions - between the kinematical constraints in Hilbert space, and events in everyday three-dimensional physical space.

With respect to option 3: Pitowsky explicitly makes this claim, saying in [15]

What is real in the quantum world? Firstly, there are objects - particles about which the theory speaks - which are identified by a set of parameters that involve no uncertainty, and can be recorded in all circumstances and thus persist through time and context. [15]

There are two problems with this, however. The first is that it seems to be in direct contradiction to other claims made elsewhere in the course of the argument. For 
example, he argues that the structure in quantum theory that represents elements of reality is the lattice of closed subspaces of Hilbert space, which he identifies as a space of possible events. Secondly, the correspondence problem described above becomes more acute in this case. Since the wave function is not part of the ontology, and therefore does not act on the material particles in any way, it is difficult to see how constraints on correlations in an abstract event space can cause material particles in three-dimensional physical space to behave in particular ways such that the disallowed correlations do not obtain. In any case, the explicit talk of physical particles underlying the QIT interpretation is absent in its fully developed form in [9].

With respect to option 4: The statement of the virtues of the QIT interpretation in [9] includes the following passage:

The possibility of a dynamical analysis of measurement processes consistent with the Hilbert space kinematic constraints justifies the informationtheoretic interpretation of quantum mechanics as realist and not merely a predictive instrument for updating probabilities. [9]

The "possibility of a dynamical analysis of measurement" to which they are referring is the existence of the dynamical explanations of measurements available to Bohmian Mechanics, GRW, and Everett. These are seen as demonstrative of the fact that the quantum dynamics is consistent with the kinematical constraints encoded in the Hilbert space structure. Any physical evolution can be so dynamically explained. What QIT takes this to mean is that the correlations required by the Hilbert space can arise, through the process of decoherence, from the quantum dynamics. But the ontological commitments of these interpretations are not thought to be indicative of a more fundamental formulation of quantum theory. That is to say, any explanation that can be given about the outcome of a particular experiment by reference to the ontology and the dynamical evolution of the states of the system over time, is not formulated in the most fundamental version of the theory.

The condition in PO that any candidate fundamental physical theory be formulated in terms of the material entities in three-dimensional space, and the dynamics describing their behavior, entails that any theory consistent with PO would be considered a non-fundamental formulation by QIT.

So what is the ontology of QIT? Bub and Pitowsky address this question by providing an explanation for what elements of reality supply the supervenience base for macroscopic objects.

On the information-theoretic interpretation, no assumption is made about the fundamental 'stuff' of the universe. So, one might ask, what do tigers supervene on? [...] In the case of the information-theoretic 
interpretation, the 'supervenience base' is provided by the dynamical analysis: tigers supervene on events defining a two-valued homomorphism in the emergent Boolean algebra. [9]

They go on to explain

The dynamics does not describe the (deterministic or indeterministic) evolution of the two-valued homomorphism on which tigers supervene to a new two-valued homomorphism (as in the evolution of a classical state). Rather, the dynamics leads to the relative stability of certain event structures at the macrolevel associated with the familiar macrosystems of our experience, and to an emergent effectively classical probability space whose atomic events are correlations between events associated with these macrosystems and microevents. [9]

There is no commitment to an underlying ontology in QIT. The dynamical equations of motion are not understood as evolving physical systems in particular states into other particular states. Rather, they evolve the "whole structure of events with probabilisitic correlations in Hilbert space" [9]. The theory is most fundamentally formulated in terms of the constraints on correlations of events in an abstract space. The theory is not fundamentally about the behavior of a particular ontology that constitutes all matter in the physical world. In this sense, QIT fails to qualify as a candidate fundamental physical theory according to PO.

However, it is interesting to note that QIT can be seen as characterizing the features that all quantum theories (in the PO sense) share. That is to say, that which makes the various quantum PO theories quantum, is their shared structure of possible events. Each offers a different explanation for why that event space is the correct one - an explanation based on the existence of a particular primitive ontology and a story about the dynamics. But QIT can be understood as offering an information-theoretic way of thinking about the shared features of all candidate fundamental formulations of quantum mechanics.

\section{References}

[1] Allori, V.: On the metaphysics of quantum mechanics. In: S. Le Bihan (ed.) Precis de la Philosophie de la Physique: d'aujourd'hui à demain. Vuibert (2013)

[2] Allori, V.: Primitive ontology and the structure of fundamental physical theories. In: A. Ney, D.Z. Albert (eds.) The Wave Function. Oxford University Press, Oxford, UK (2013) 
[3] Allori, V., Goldstein, S., Tumulka, R., Zanghì, N.: On the common structure of Bohmian Mechanics and the Ghirardi-Rimini-Weber theory. British Journal for the Philosophy of Science 59, 353-389 (2008)

[4] Allori, V., Goldstein, S., Tumulka, R., Zanghì, N.: Predictions and primitive ontology in quantum foundations: A study of examples. British Journal for the Philosophy of Science 65(2), 323-352 (2014)

[5] Bell, J.S.: Beables for quantum field theory. In: Speakable and Unspeakable in Quantum Mechanics. Cambridge University Press, Cambridge, UK (1987)

[6] Bell, J.S.: The theory of local beables. In: Speakable and Unspeakable in Quantum Mechanics. Cambridge University Press, Cambridge, UK (1987)

[7] Bub, J.: Why the quantum? Studies in the History and Philosophy of Modern Physics 35, 241-266 (2004)

[8] Bub, J.: Quantum mechanics is about quantum information. Foundations of Physics 35(4), 541-560 (2005)

[9] Bub, J., Pitowsky, I.: Two dogmas about quantum mechanics. In: S. Saunders, J. Barrett, A. Kent, D. Wallace (eds.) Many Worlds?, pp. 433-459. Oxford University Press, Oxford, UK (2010)

[10] Clifton, R., Bub, J., Halvorson, H.: Characterizing quantum theory in terms of information-theoretic constriants. Foundations of Physics 33(11), 1561-91 (2003)

[11] Dürr, D., Goldstein, S., Zanghì, N.: Quantum equilibrium and the origin of absolute uncertainty. Journal of Statistical Physics 67, 843-907 (1992)

[12] Goldstein, S.: Quantum theory without observers. Physics Today (1998)

[13] Monton, B.: Wave function ontology. Synthese 130, 265-77 (2002)

[14] Monton, B.: Against 3n-dimensional space. In: A. Ney, D.Z. Albert (eds.) The Wave Function, pp. 154-167. Oxford University Press (2013)

[15] Pitowsky, I.: Quantum mechanics as a theory of probability. In: W. Demopoulos, I. Pitowsky (eds.) Physical Theory and Its Interpretation, The Western Ontario Series in Philosophy of Science, pp. 213-240. Springer (2006)

[16] Wallace, D.: The Emergent Multiverse: Quantum Theory According to the Everett Interpretation. Oxford University Press, Oxford, UK (2012) 